\title{
A New Method of Tracking Motion Human Based on Multiple Pyroelectric Sensors
}

\author{
Hui Zeng ${ }^{1}$, Xueming $\mathrm{Hu}^{1}$, Nan Zhang ${ }^{1}$, Ji Xiong ${ }^{2, a}$ \\ ${ }^{1}$ Wuhan Maritime Communication Research Institute, Wuhan, China \\ ${ }^{2}$ School of Information Engineering, Wuhan Technology and Business University, Wuhan, China
}

Keywords: pyroelectric infrared (PIR) sensor, human detection, direction detection, distance identification, fusion algorithm.

\begin{abstract}
Pyroelectric infrared (PIR) sensor is low-cost, low-power small component commonly used only to trigger alarm in presence of humans or moving objects. PIR sensors are widely used as a simple, but reliable. In this paper, we present a novel pyroelectric sensor node, feature extraction method and human pyroelectric information fusion method to identify direction of multi-human motion, number of people passing and distance between the PIR sensor and people in an array of pyroelectric sensors. The pyroelectric sensor node is composed of two kinds of pyroelectric sensor modules. Each module has different function, so we can fuse the different function to accurately track human target. The approach has low computational requirement, so it is well-suited for limited-resources devices. Through the experiment, we can get the correct detection rate of the direction and number of people is more than $100 \%$, the correct identification rate of the path is more than $78 \%$.
\end{abstract}

\section{Introduction}

Human detection, identification and motion tracking have much attention in fields as surveillance, security application and smart environments. Conventional tracking techniques use cameras to extract features such as number of people, position and direction ${ }^{1}$, but these techniques need process large amounts of data. Even if these solutions are accurate, they have high cost and require significant infrastructure.

The pyroelectric infrared (PIR) sensor has high performance for IR detection, and it does not need to use special and expensive cooling equipment like a photon-type detector. In recent years, pyroelectric devices have been extensively used in the people's livelihood, such as intruder detecting and wisely light controlling. Several studies have touched on other applications, such as monitoring the flow in traffic, collecting people's information, etc. Most of these usages belong to qualitative applications.

Recently, researchers have been working on detecting movement direction, velocity and counting people entering or leaving at the entrance of a room or building using PIR sensors. For example, Kaushik had investigated the characteristics and spatial sensitivity of PIR detector through experiments. Finding that the detector output had an evident relationship with the velocity and vertical

${ }^{\mathrm{a} C}$ Corresponding author :bear-xiongji@163.com 
distance of movements, they pointed out that a PIR system can be used to monitor the occupancy pattern of elderly people ${ }^{2}$.

Gopinathan $^{3}$ et al. designed code apertures to modulate the visibility between source and measurement spaces and as reference structures cooperated with four PIR sensor to develop a system able to detect the path of a single people moving in an area. Shankar ${ }^{4}$ et al. a low cost sensor cluster was used to extract velocity as well as the path of a single people. Honda et al. installed 40 infrared sensors at doors, gateways of the laboratory to extract individual human behaviors from long-term data $^{5}$. Hashimoto et al. presented a people count system, which consisted of 1-dimensional 8-element array detectors fabricated, IR-transparent lens, and oscillating mechanical chopper. The people count system could get $99 \%$ recognition accuracy in moving direction and $95 \%$ recognition accuracy of number of passerby ${ }^{6}$.

Zappi et al. presented human tracking system for detecting the direction ${ }^{7}$ and distinguishing the distance intervals between human target and PIR sensor ${ }^{8}$, the system consisted of two PIR sensors, which be placed in a hallway with facing each other. They used Support Vector Machines and KNearest Neighbor (k-NN) algorithm to classify collection of experimental data, obtained 100\% recognition accuracy in direction of movement and 83.49-95.35\% recognition accuracy in distance interval.

In this paper, we present a novel method to track human using our system in indoor. We extract different features from the PIR data collected, and then fusion of multi-sensing features to distinguish the direction of multiple motion humans, recognize the number of multiple motion humans and the distance between the PIR node and motion human ${ }^{9}$. As a result of amount of calculation of our method is relative small and accuracy, if equipped with low-cost, low-power devices, it is well suited for implementation on wireless sensor network (WSN). At the same time, we have developed a data collection apparatus, which is consisting of two PIR sensors with Fresnel lenses and different masks. This equipment has special field of views. When people walk in the FoV(Field of View), our apparatus can collect data from human thermal infrared, then calculate the distance of human from PIR sensor and other features based on the novel method.

Our approach shows that the raw data is captured from a PIR node in conjunction with modified mask. It is possible to detect the five distances between walking human and PIR node with 78\%-94\% recognition accuracy in the range of $1.2 \mathrm{~m}$ to $6.6 \mathrm{~m}$, the correct detection rate of movement direction is $100 \%$ and correct detection rate of the number of human is $100 \%$, So we can now conclude with confidence that the proposed method can provide greater accurate to sense and locate human target in indoor, such as healthcare and larger company.

\section{The analysis of data and theory model}

The PIR sensor is suitable for working at the frequency from 0.1 to $100 \mathrm{~Hz}$. Because the PIR sensor responds to the rate of change of temperature $d T / d t$, it is suitable to measure pulse type radiation power.

The objectives of our PIR sensor module are to recognize the number of people, direction of movement and movement path through the sensor module in different situations. Detecting the number of peaks at the output of the sensor module, this can get number of people. This task can be achieved quickly while the people walks through. Therefore as soon as the end of an event is detected the sensor node can send its features at the coordinator which, by means of few additional controls on data, can detect the number of individuals and direction of their movement ${ }^{10}$.

\subsection{The analysis of A-type PIR sensor module}

The A-type PIR module consists of PIR sensor, Fresnel Lens and mask. Different masks have different sensing aperture. The aperture of A-type PIR module is designed enough small (about 2mm), which will form narrow the FOV of the module. And that has two positive effects: 
1) helping in distinguishing different people moving in sequence and back and forth

2) Each passage produces only a complete peak which can judge motion direction.

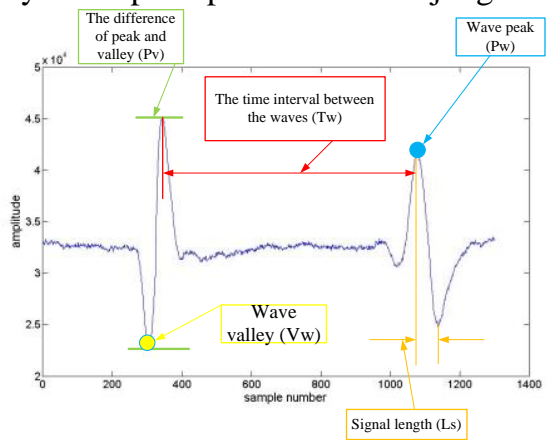

Figure 1. A type of PIR module sensing waveform

From the Fig.1, we can see the pulsed sinusoidal signal. With the increasing of the movement distance between human target and PIR node, the $\boldsymbol{L} \boldsymbol{s}$ is increasing, the difference of wave peak and valley $(\boldsymbol{P} \boldsymbol{v})$ is reducing, so we can get.

$$
\Gamma=\frac{\Delta P v}{\Delta L s}
$$

According to processed waveform of the Fig. 1, we can obtain lots of different $\Gamma$ parameters, at the same time the fitting method is used to build a distance model which will be built by multidimensional variable, as shown in Eq. (2).

$$
y=m_{0}+m_{1} x_{1}+m_{2} x_{2}+\ldots .+m_{n} x_{n}+\varepsilon
$$

We assume that there are $\mathbf{M}$ groups experiment data. From the multidimensional linear regression model, we can get the following expressions.

$$
\left\{\begin{array}{c}
y_{1}=m_{0}+m_{1} x_{11}+m_{2} x_{21}+\ldots .+m_{n} x_{n 1}+\varepsilon_{1} \\
y_{2}=m_{0}+m_{1} x_{12}+m_{2} x_{22}+\ldots .+m_{n} x_{n 2}+\varepsilon_{2} \\
\vdots \\
y_{m}=m_{0}+m_{1} x_{1 m}+m_{2} x_{2 m}+\ldots .+m_{n} x_{n m}+\varepsilon_{m}
\end{array}\right.
$$

$\mathbf{Y}$ is defined as the measurement matrix, and $\mathbf{X}$ is defined as the observation matrix. It can be expressed as a matrix.

$$
Y=X M+\varepsilon
$$

In order to obtain the desired fitting function, we need to build a target function.

$$
J=\|Y-X M\|_{\min }
$$

When $\frac{\partial J}{\partial M}=0$,we can get $\mathbf{M}$.

$$
M=\left(X^{T} X\right)^{-1} X^{T} Y
$$

We use 30 groups data to fit the mathematical model, and form a fitted region, then the median algorithm is used to calculate median values of the fitted region. Finally, we can get the mathematical model $\mathbf{y}$.

\subsection{The analysis of B-type PIR sensor module}

The aperture of signal modulation mask is designed to be $4 \mathrm{~mm}$. The main effect has two aspects:

1). The pyroelectric sensor can obtain more human body radiation energy.

2). The thermal infrared energy of multi-people in closer distance can be detected simultaneously. 
When two peoples cross the sensing region and the two complete waveforms will be formed by the PIR sensor. Th represents critical distances parameter for different waveforms, and $d$ represents distance between two peoples.

1). When two human targets walk into the sensing region, if the distance between two people is $d<T h$, will produce two successive waves, the peaks and valleys of the wave are alternate.

2). If the distance between two peoples is $d>T h$, the peaks and valleys of the wave will be superimposed on each other.

\section{Proof :}

We assume that there are six different sensing models, as shown in Fig.2.

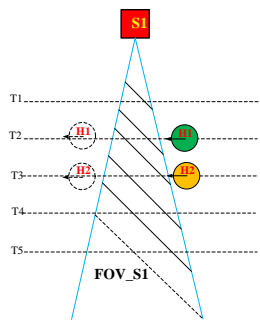

(a)

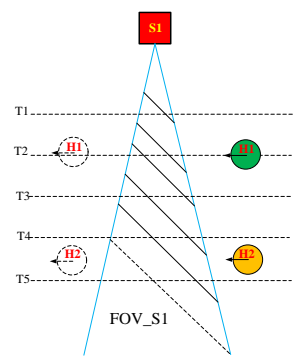

(d)

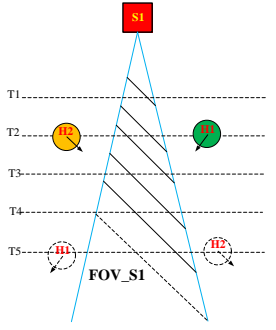

(b)

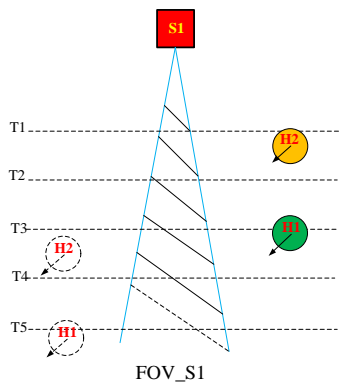

(e)

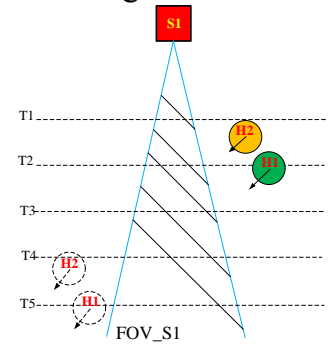

(c)

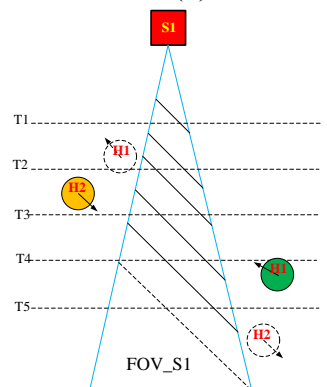

(f)

Figure 2. Six different sensing models for two human targets detection

Fig .2(a) describes two closer human targets in horizontal distance, and at the same time, they cross the sensing region. Fig .2(b) describes two human targets that cross the region about 90 degrees in the direction of intersected moving. Fig.2(c) describes two closer human targets that cross the region on the bias. Fig .2(d) describes two human targets that cross the region in horizontal line and the distance between each other is larger. Fig .2(e) describes two closer human targets that cross the region on the bias and the distance between each other is larger. Fig .2(f) describes two closer human targets that cross the region in opposite direction.

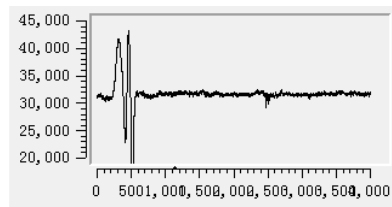

(a)

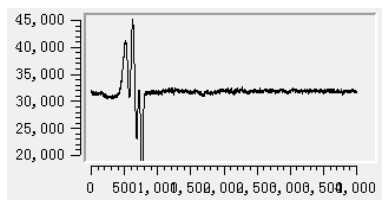

(d)

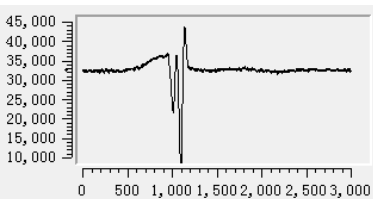

(b)

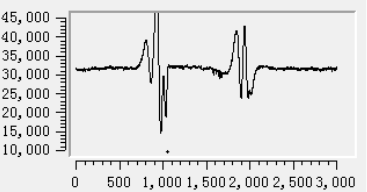

(e)

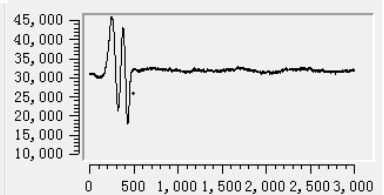

(c)

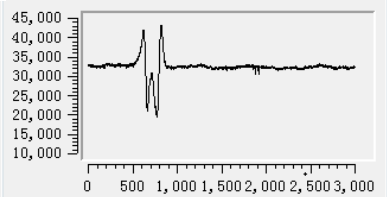

(f)

Figure 3. Measurement data of six different sensing models 
1). Due to the distance between two peoples is less than the threshold, the sensing mode of two peoples is similar to single people sensing mode. Base on the different position between the two peoples, in the Fig .3(a), the first wave peak is smaller than the second wave peak.

2). In the Fig .3(b), due to the movement direction of two peoples is opposite, when they move into the sensing region at the same time, the sensing vector signal of the two peoples is superimposed, the superimposed signal is nonstandard and disorder.

3). In the Fig .3(c), because the distance between two peoples is much closed, the sensing mode of two peoples is similar to the single people sensing mode. Because people $\mathrm{H} 2$ enters the sensing region at first, so in the Fig .3(c), the parameter Ls of the first wave peak is larger than the second wave.

4). In the Fig .3(d), compared to Fig .3(a), the distance between two peoples is larger than the threshold, when people $\mathrm{H} 2$ enters the sensing region, the first wave peak will form, then the people $\mathrm{H} 1$ enters the sensing region, and the second wave peak will form, due to the distance between the two peoples is different from the Fig .3(a), the position of continuous wave peak is different.

5). In the Fig .3(e), the wave peak and valley is similar to the Fig .3(d).

In the Fig .3(f), due to the movement direction of two peoples is opposite, and the people H2 enters region at first, then the people $\mathrm{Hl}$ also will enter the region. So the two waves are opposite

\section{Fusion algorithm}

A-type PIR sensor module has better ability in direction distinction and can identify the number of human target. When lots of peoples walk in the sensing region and the distance $\mathrm{d}$ between peoples is less than the threshold $T h$, it will have limited recognition ability. B-type PIR sensor module can identify the direction and the number of multi-human.

So fusion of the A-type and the B-type PIR sensor module, will can improve the efficiency and validity in the complex environment. The fusion rules are as follows

1) When the A-type PIR module collects a complete pulse sine wave, however, the B-type PIR module collects two continuous pulse sine waves, according to the number of wave peak, we can estimate the number of human target, and according to the characteristics of the waveform we can judge the moving direction of the human target.

2) When the A-type PIR module collects lots of pulse sine waves at frequent intervals, we can judge that lots of human targets cross the PIR module in a sequence mode.

3) When the B-type PIR module collects two continuous pulse sine waves and the A-type PIR module collects two wave peaks, we can judge that two peoples in sensing region and the distance between the two peoples is larger than the threshold.

\section{Experimental tests and verification}

\subsection{Judgment test of human moving direction and number}

In order to prove our fusion theory, we carry out several experiments.

1) Direction test of single human motion

Table 1. The test of human motion direction

\begin{tabular}{|c|c|c|c|c|}
\hline Number of testers & Experimental model & Experiment number & Accurate direction & Accurate numbers \\
\hline 1 & Single people & 400 & $400 / 400(100 \%)$ & $400 / 400(100 \%)$ \\
\hline 4 & Single people & $4 * 100$ & $400 / 400(100 \%)$ & $400 / 400(100 \%)$ \\
\hline
\end{tabular}

2) Direction test of Multiple human targets 
There are four couples human target, and they walk into the sensing region in the horizontal and 45 degrees direction. There are six kinds of motions mode as shown in Figure 3.

Table 2. Experiment results

\begin{tabular}{|c|c|c|c|l|}
\hline Number of couples & The experimental model & Experiment number & Accurate direction & Accurate numbers \\
\hline 4 & Figure 3(a) & $4 * 20$ & $74 / 80$ & $78 / 80(97.5 \%)$ \\
\hline 4 & Figure 3(b) & $4 * 20$ & $54 / 80$ & $66 / 80(82.5 \%)$ \\
\hline 4 & Figure 3(c) & $4 * 20$ & $67 / 80$ & $75 / 80(93.7 \%)$ \\
\hline 4 & Figure 3(d) & $4 * 20$ & $71 / 80$ & $80 / 80(100 \%)$ \\
\hline 4 & Figure 3(e) & $4 * 20$ & $62 / 80$ & $71 / 80(88.75 \%)$ \\
\hline 4 & Figure 3(f) & $4 * 20$ & $72 / 80$ & $63 / 80(78.75 \%)$ \\
\hline
\end{tabular}

\subsection{Identification test of human moving path}

Three human targets walk along the ten kinds of different paths, and they carry out twenty times experiments respectively. At first, the human target walks ten times in five different paths, including $1.2 \mathrm{~m}, 2.4 \mathrm{~m}, 3.6 \mathrm{~m}, 4.8 \mathrm{~m}$ and $6.0 \mathrm{~m}$, then in other five different paths ten times, including $1.8 \mathrm{~m}, 3.0 \mathrm{~m}, 4.2 \mathrm{~m}, 5.4 \mathrm{~m}$ and $6.6 \mathrm{~m}$. We assume that the distance of $1.2 \mathrm{~m}, 2.4 \mathrm{~m}, 3.6 \mathrm{~m}, 4.8 \mathrm{~m}$ and $6.0 \mathrm{~m}$ is $M$ type, the distance $1.8 \mathrm{~m}, 3.0 \mathrm{~m}, 4.2 \mathrm{~m}, 5.4 \mathrm{~m}$ and $6.6 \mathrm{~m}$ is $N$ type. We use multidimensional fitting algorithm to fit the $N$ type data of the first human target, so the distance relationship mathematical model of the first human target is got. After that, the $M$ type data is fed into the mathematical model and can get validation data.

$$
y=9.19 \times 10^{-6} x^{2}-1.68 \times 10^{-2} x+8.09
$$

Table 3. The $N$ type fitting model of the first human target

\begin{tabular}{|c|c|c|c|}
\hline Observed value of $M$ type $(\Gamma$ value $)$ & Distance $(m)$ & The validation data & Difference value \\
\hline 604 & 1.2 & & \\
\hline 461 & 2.4 & 477 & 16 \\
\hline 322 & 3.6 & 356 & 34 \\
\hline 216 & 4.8 & 245 & 29 \\
\hline 144 & 6.0 & 149 & 5 \\
\hline
\end{tabular}

Table 4. The $M$ type fitting model of the first human target

\begin{tabular}{|c|c|c|c|}
\hline Observed value of $N$ type $\left(\Gamma_{\text {value }}\right)$ & Distance $(\mathrm{m})$ & The validation data & Difference value \\
\hline 538 & 1.8 & 536 & 2 \\
\hline 415 & 3.0 & 387 & 28 \\
\hline 299 & 4.2 & 264 & 35 \\
\hline 192 & 5.4 & 175 & 17 \\
\hline 115 & 6.6 & & \\
\hline
\end{tabular}

The distance relationship mathematical model of the second human target:

$$
y=8.1 \times 10^{-6} x^{2}-1.70 \times 10^{-2} x+8.6
$$

The distance relationship mathematical model of the third human target:

$$
y=8.4 \times 10^{-6} x^{2}-1.73 \times 10^{-2} x+8.3
$$

From the mathematical models of three human targets, we can see that the coefficients of mathematical models are very similar. Difference value of different distances is less than fifty. So through the analysis of the mathematical models, the distance relationship between the PIR sensor and moving human target can be obtained, as follow mathematical model. 


$$
\mathrm{y}= \begin{cases}1.2 m \sim 2.4 m & \Gamma \subset[500,650] \\ 2.4 m \sim 3.6 m & \Gamma \subset[400,500] \\ 3.6 m \sim 4.8 m & \Gamma \subset[250,400] \\ 4.8 m \sim 6.0 m & \Gamma \subset[170,250] \\ 6.0 m \sim 6.6 m & \Gamma \subset[100,150]\end{cases}
$$

In order to verify the mathematical models of path identification, we invite five different peoples, collect five different path data, and prove the mathematical model.

Table 5. The result of path experiment

\begin{tabular}{|c|c|c|c|}
\hline Number of testers & Distance interval $(\mathrm{m})$ & Experiment number & Correct recognition rate \\
\hline \multirow{5}{*}{5} & $1.2 \mathrm{~m}$ to $2.4 \mathrm{~m}$ & $10 * 5$ & $47 / 50(94 \%)$ \\
\cline { 2 - 4 } & $2.4 \mathrm{~m}$ to $3.6 \mathrm{~m}$ & $10 * 5$ & $41 / 50(82 \%)$ \\
\cline { 2 - 4 } & $3.6 \mathrm{~m}$ to $4.8 \mathrm{~m}$ & $10 * 5$ & $44 / 50(88 \%)$ \\
\cline { 2 - 4 } & $4.8 \mathrm{~m}$ to $6.0 \mathrm{~m}$ & $10 * 5$ & $41 / 50(82 \%)$ \\
\cline { 2 - 4 } & $6.0 \mathrm{~m}$ to $6.6 \mathrm{~m}$ & $10 * 5$ & $39 / 50(78 \%)$ \\
\hline
\end{tabular}

\section{Conclusions}

In this paper we introduced an approach to detect moving direction of multi-human targets, the number of people passing and distance between the human and PIR sensor by means of a set of two PIR detectors. The detectors are located along a hallway, which can collect the features from the two PIR sensor modules and it can infer direction of movement, number of people and the path.

\section{References}

1. R. Bodor, B.Jackson and N. Papanikolopoulos. Visionbased human tracking and activity recognition. In: 11th Mediterranean Conf. on Control and Automation, 2003

2. A. Kaushik and B. Celler. Characterization of Passive Infrared Sensors For Monitoring Occupancy Pattern. In: Proceedings of 28th Annual International Conference. On the IEEE Engineering in Medicine and Biology Society (EMBS), September 2006, p. 5257-5260.

3. U. Gopinathan, D. Brady and N. Pitsianis. Coded apertures for efficient pyroelectric motion tracking. Opt. Exp.Vol.10(2003), p. 2142-2152.

4. M. Shankar, J. B. Burchett, Q. Hao, B. D. Guenther and D. J. Brady. Human-tracking systems using pyroelectric infrared detectors. Opt. Eng. Vol.45(2006), p. 106401-1-106401-10.

5. S. Honda, K.I. Fukui, K. Moriyama, S. Kurihara and M. Numao. Extracting human behaviors with infrared sensor network. In: Proc. 4rd Int. Conf. on Networked Sensing Systems, Braunschweig, Germany, June 2007, p. 122-125.

6. K. Hashimoto, K. Morinaka, N. Yoshiike, C. Kawaguchi and S. Matsueda. People count system using multi-sensing application. In: Proc. Int. Conf. on 'Solid State Sensors Actuat', Jun 1997, p.1291-1294.

7. P. Zappi, E. Farella and L. Benini. Tracking motion direction and distance with pyroelectric IR sensors. IEEE Sensors J. Vol.10(2010), p. 1486-1494.

8. J. S. Fang, Q. Hao, D. J. Brady, M. Shankar, B. D. Guenther and N. P. Pitsianis. Path-dependent human identification using a pyroelectric infrared sensor and fresnel lens arrays. Opt. Exp. Vol. 14(2006), p. 609-624.

9. X. H.Lv and Y.X.Liu. Design of human motion detection and tracking robot based on pyroelectric infrared sensor. In: Proceedings of the IEEE Int. Conf.on the 8th world congress on intelligent control and automation, 2010, p.3737-3740.

10. J. Lu, J. Q. Gong and Q. Han. Multi-agent based wireless pyroelectric infrared sensor networks for multi-human tracking and self-calibration. In: Proceedings of the IEEE Int. Conference on sensors, 2013, p.1-4. 